\title{
Décadrages Décadrages
}

cinéma, à travers champs Cinéma, à travers champs

4-5 | 2005

David Lynch

\section{L'éclatement du personnage chez Lynch.}

Les « mondes possibles » dans Lost Highway et Mulholland Drive

Alain Boillat

\section{CpenEdition}

\section{Journals}

Édition électronique

URL : https://journals.openedition.org/decadrages/577

DOI : $10.4000 /$ decadrages.577

ISSN : 2297-5977

Éditeur

Association Décadrages

\section{Édition imprimée}

Date de publication : 10 avril 2005

Pagination : 48-61

ISBN : 978-2-9700582-1-2

ISSN : 2235-7823

Référence électronique

Alain Boillat, «L'éclatement du personnage chez Lynch. », Décadrages [En ligne], 4-5 | 2005, mis en

ligne le 29 avril 2013, consulté le 27 mars 2022. URL : http://journals.openedition.org/decadrages/577 ; DOI : https://doi.org/10.4000/decadrages.577

(B) Décadrages 


\section{L'éclatement du personnage chez Lynch}

\section{Les "mondes possibles" dans Lost Highway et}

\section{Mulholland Drive}

1 Comme le note Youri Deschamps: "La sortie du dernier film de Lynch fut accompagnée d'une véritable ivresse de commentaires, que les visions multiples sont rarement venues tarir. Lost Highway avait déjà suscité pareil engouement actif; l'opacité du mystère et la déroute sensorielle avaient trouvé une réponse adéquate dans la ferveur et l'implication du public" ("Meraviglia et complexe de Corvick", in Eclipses, $n^{\circ} 34$, p. 4).

2 L'avertissement sur lequel s'ouvre ce film d'une vingtaine de minutes est révélateur d'un respect certain pour la pureté quasi virginale de la première vision du film et du sens caché de celui-ci, puisqu'on fait savoir au spectateur que "certaines scènes de ce documentaire peuvent déflorer les secrets de Mulholland Drive".

3 Ces indications qui se présentent sous la forme de questions ou de conseils ("soyez attentifs à...") ont été reprises pour la partie intérieure de la jaquette du DVD français, également édité par Canal+. Si le commentaire du reportage répond de façon fort convaincante à certaines interrogations imaginées par Lynch pour orienter son public, il tend parfois à laisser dans l'ombre quelques éléments appartenant à des lignes narratives secondaires dont la prise en compte pourrait remettre en cause son modèle d'analyse. Ainsi les auteurs du commentaire répondent-ils au dernier point ("Où est tante Ruth?") en citant deux affirmations données verbalement dans le film: tante Ruth est sur un tournage au Canada (dans "le rêve") / elle est décédée (dans "la réalité"). Ils ne font par contre aucune mention du plan où tante Ruth pénètre dans la chambre où Betty et Rita viennent de disparaître, observe la pièce vide, éteint puis s'éloigne. Le retour au quotidien induit par ce plan suggère que Diane aurait également pu ne pas recevoir l'argent de l'héritage, et donc ne pas être la commanditaire du meurtre de Camilla.

4 Alain Bergala, "A Rachel O., qui hésite à entreprendre des études de cinéma", in Cahiers du cinéma, n 593, septembre 2004, p. 9.

\section{par Alain Boillat}

Rares sont les films qui, comme Mulholland Drive (2001), provoquèrent auprès du public, dans la presse et dans les ouvrages spécialisés une telle soif d'interprétation d'une structure narrative. Dans ce film, Lynch renoue après le récit très linéaire de Une histoire simple (1999) avec les ambiguïtés narratives de Lost Highway (1997) 1. La chaîne Canal+, coproductrice de Mulholland Drive, a même proposé à l'occasion de la première diffusion du film sur ses ondes un court métrage explicatif (Retour à Mulholland Drive, réalisé en 2003 par Philippe Rouyer, critique à Positif) censé livrer les clés requises pour une «bonne» compréhension de l'œuvre ${ }^{2}$. L'interprétation proposée dans ce reportage se base notamment sur les indices livrés dans la presse par le Mâ̂tre lui-même une semaine après la sortie du film ${ }^{3}$.

A force d'être constamment affichée dans le film et entretenue par les discours qui en ont accompagné la sortie, cette prétendue complexité pourrait sembler lassante, voire surfaite. On pourrait être tenté d'y voir une stratégie commerciale consistant à produire un film que les spectateurs doivent revoir pour lui extirper des «secrets» dont l'Auteur souligne l'importance tout en se retranchant, pour éviter toute explication, derrière le mythe de l'inspiration créatrice. Toutefois, Lost Highway et Mulholland Drive résistent aux assauts répétés de cette herméneutique effrénée. Ils n'en sont pas moins, au vu de leurs particularités narratives, emblématiques du «bon objet» académique. Alain Bergala prenait récemment comme exemples canoniques d'étudiants entreprenant un travail universitaire ceux qui "vont entrer pour un $137^{\mathrm{e}}$ sujet exaltant sur la structure de la narration chez Lynch» $\mathbf{4}$.

La narratologie et les films de Lynch font en effet (trop) bon ménage. On a presque l'impression que l'analyste se trouve enfermé dans une grille de lecture déterminée par les films eux-mêmes qui l'amènent 
à «découvrir» des principes auxquels ils obéissent explicitement, à l'instar du sémiologue qui croit fournir une description originale du fonctionnement d'un message publicitaire alors qu'il recourt exactement aux mêmes outils théoriques qui ont été utilisés en amont par les concepteurs du produit. Néanmoins, je crois qu'une énième étude narratologique des films de Lynch peut s'avérer d'un certain intérêt dans la mesure où il n'est pas aisé d'épuiser les interrogations soulevées par leur agencement narratif. Il y a du «jeu» dans les rouages huilés du récit lynchien qui procède d'une combinatoire ludique, voire aléatoire. S’il est vrai que Lynch semble vouloir "piéger les tentatives et les tentations d'une interprétation univoque", cet aspect de sa démarche ne soulève pas, à mon avis, "le problème de la pertinence, voire de la possibilité d'un commentaire explicatif ${ }^{\mathbf{5}}$, mais ouvre au contraire une brèche salutaire dans une entreprise a priori balisée.

Plutôt que de concevoir Lost Highway et Mulholland Drive, à l'instar de la plupart de leurs exégètes - dont les propositions n'en sont pas moins éclairantes à de nombreux égards -, comme la juxtaposition d'une partie "réelle» (les vingt dernières minutes de Mulholland Drive, les cinquante premières de Lost Highway) et d'une partie "rêvée », je préfère éviter d'épuiser le potentiel énigmatique de ces films en renonçant à une interprétation livrée "clé en main» $\mathbf{6}$, forcément trop rassurante et réductrice. La dichotomie rêve/réalité me semble insuffisante pour aborder de tels films éclatés qui rendent caducs de nombreux principes de la narration traditionnelle. En outre, le rétablissement d'une temporalité diégétique supposée (notamment en ce qui concerne les deux flash back de la seconde partie de Mulholland Drive) conduit à une linéarisation qui occulte la question du positionnement du spectateur durant le visionnement du film, et de ce fait certains procédés de déstabilisation auxquels il est confronté. Par conséquent, il me semble plus adéquat d'opter pour un cadre d'analyse qui offre la latitude nécessaire à la prise en compte du caractère dynamique des processus mis en œuvre par les films et actualisés in fine par le spectateur. J'abonde dans le sens de Michel Chion lorsqu'il affirme que «la seule constante du cinéma de Lynch, son postulat, est l'existence de plus d'un monde» 7 . Dans cette optique, je propose de considérer le basculement dans le rêve comme un mode parmi d'autres de redistribution des données narratives, comme la mise en place d'un nouveau monde possible $\mathbf{8}$. L'économie narrative de tels films repose sur des opérations telles que la permutation, la variation, l'ajout ou la suppression ${ }^{9}$. Je la considérerai dans sa dimension cyclique en mettant l'accent sur les modalités de circulation des objets et de certains traits physiques et psychologiques des personnages. Lost Highway
5 Diane Arnaud, "Lost Highway de David Lynch. La mort à l'œuvre. L'œuvre à mort? " in Murielle Gagnebin (sous la dir.), Cinéma et inconscient, Editions Champ Vallon, Seyssel, 2001, p. 289.

6 Cette conception binaire réalité/rêve est défendue notamment dans Retour à Mulholland Drive, mais aussi dans la plaquette de Georgy Katzarov, L'invention du rêveur, Editions Inventaire/Invention, Paris, 2002.

7 Michel Chion, David Lynch, Editions des Cahiers du Cinéma, Paris, 2001, p. 196.

$8 \mathrm{~A}$ cet égard, les deux récits de Lynch s'inscrivent plus nettement dans les paradigmes de pensée issus de la réflexion sur les technologies contemporaines du "virtuel" que leur univers proprement dit ne pourrait le laisser paraître, marqué qu'il est par une iconographie rétro ou recourant à des stéréotypes de l'Age d'Or de Hollywood.

9 II n'est en ce sens pas étonnant que Lynch se soit intéressé à la réalisation de séries, la régularité d'une telle diffusion télévisuelle constituant un lieu privilégié d'expansion des possibles, tout comme le recours à d'autres moyens d'expression (la musique, la bande dessinée, la peinture). 
10 Cette séquence fait suite à celle où Betty rencontre un vif succès lors d'un autre casting durant lequel elle séduit un acteur d'âge mûr. Or, ce casting-là est effectué pour un cinéaste appelé Bob Brooker qui sera identifié lors de la soirée dans la villa comme étant l'auteur de L'histoire de Sylvia North, film pour lequel Adam auditionne dans cette séquence. Les castings, moments charnières dans la carrière de l'actrice, constituent donc de potentielles bifurcations narratives.

11 Pour plus de détails sur cette chanson, voir l'article "Silencio: Lynch tourne!" dans la présente livraison.

12 Significativement, la première phrase que Betty prononce à son arrivée à Los Angeles, alors qu'elle contemple, ébahie, la métropole, est "I can't believe it!". Dans son sens littéral, cette expression suggère que la perception de ce personnage-spectateur est avant tout un phénomène de croyance face à l'illusion hollywoodienne et cinématographique.

13 Le film met principalement l'accent sur les personnages féminins, la victimisation du cinéaste Adam Kersher tournant rapidement à une représentation grotesque. Alors que les angoisses de Rita, puis les frustrations de Diane sont prises au sérieux, les démêlées du réalisateur avec les protagonistes masculins qui incarnent le pouvoir décisionnel confèrent au film une tonalité comique que souligne l'utilisation de figures cinématographiques caricaturales (le cow-boy, le mafieux, l'amant, l'homme de main). débute et se termine par un rapide travelling avant sur une route, l'horizon incertain des possibles s'évanouissant dans la nuit, puisqu'on ne sait pas quel visage prendra le héros après les dernières convulsions qui l'assaillent. Dans Mulholland Drive, le cadavre décomposé de Diane ne peut pas être celui de la rêveuse étant donné que, si tel était le cas, elle ne serait plus capable de rêver; toutefois, sa position est strictement identique à celle qui sera la sienne après son suicide. Ce corps étendu, pivot du film, est à la fois le sien et celui d'une autre (comme en témoignent les variations sur la couleur de la nuisette) dont elle imite sans le savoir le parcours sentimental et professionnel. La répétition renvoie au processus de destruction de l'individu à l'œuvre au sein de "l'usine à rêves» de Hollywood, où le "rêve» n'est jamais unique et individuel, mais résulte d'une production organisée sur le modèle de l'industrie. Lorsque, à la fête dans la villa du cinéaste, Rita/Camilla embrasse sur la bouche une nouvelle venue blonde, on comprend que cette invitée est une future victime du système comme Diane, ou une autre incarnation de la femme fatale comme Camilla. D'ailleurs, Betty aurait pu faire carrière si, comme Camilla, elle avait courtisé le réalisateur Adam Kersher ${ }^{\mathbf{1 0}}$, ce qui lui aurait permis, à elle qui n'a jamais pu être la vedette de ses films, d'inverser les rôles. Cette opportunité est suggérée lorsque, durant le casting du film L'bistoire de Sylvia North, Adam se retourne et fixe Betty, un zoom avant nous rapprochant d'elle jusqu'au gros plan, alors que retentit l'air d'une chanson d'amour ${ }^{\mathbf{1 1}}$. Cette représentation stéréotypée du coup de foudre s'avérera déceptive puisqu'elle ne donnera lieu à aucun développement. La scène où se manifeste l'attirance d'Adam pour Betty ne fait qu'exposer un possible demeuré inexploité. Betty, située dans l'espace du public et des machinistes, est opposée spatialement à l'heureuse élue qui chante sur scène, l'actrice Camilla Rhodes dont Rita reprendra le nom lorsque le possible de la concurrence avec Betty/Diane sera actualisé. Or, l'actrice qui interprète cette première Camilla est également celle qui, vêtue d'une robe aux mêmes tons pastel, embrasse devant Diane la seconde Camilla lors du souper chez Adam. Si l'on s'en tient aux noms, on peut dire que Camilla «s'embrasse elle-même», la blonde de la seconde partie n’étant pas nommée. La négation homosexuelle de l'altérité atteint alors son paroxysme.

Mulholland Drive, reformulation d'un projet avorté de série télévisuelle - un mode de représentation qui demeure généralement latent dans le film, mais fait saillie çà et là -, présente une structure qui tend à reproduire la standardisation induite par la diffusion de masse. L'évolution de la candeur admirative $\mathbf{1 2}$ à la désillusion suicidaire n'est pas propre à Betty, ni même à son double, mais à toutes celles ${ }^{\mathbf{1 3}}$ qui sont 


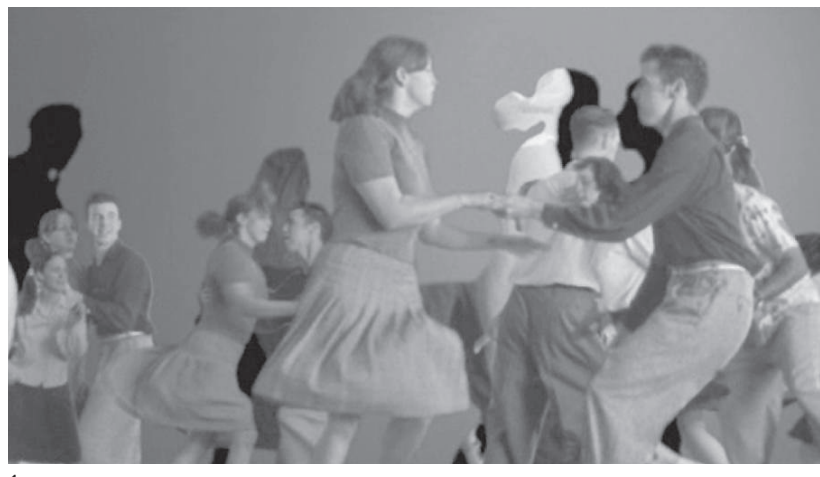

abusées par les attraits factices du milieu hollywoodien. Lorsque Betty aspire à faire carrière, elle oublie que la star est avant tout une construction de l'industrie du spectacle. Le film nous le rappelle en exhibant le caractère construit du personnage lui-même.

Le générique de Mulholland Drive exprime de façon littérale et graphique cette multiplication anonyme des êtres. On y voit des couples danser, leurs ombres stylisées découpant sur un arrière-fond mauve des surfaces noires qui laissent percevoir d'autres couples auxquels les premiers se superposent dans la fausse perspective de cette image à la planéité affichée (fig. 1). Après avoir été réduits dans un plan liminaire à d'indistinctes formes mouvantes colorées, les individus accèdent ensuite à une représentation figurative, mais continuent d'être perçus comme des surfaces géométriques qui se croisent, s'interpénètrent et s'évanouissent pour resurgir, identiques, ailleurs dans l'image. Ces couples, à la fois fondus dans l'agitation de l'ensemble et reconnaissables à certains traits vestimentaires (qui donnent lieu à des combinaisons spécifiques), sont emportés par un rythme jazzy répétitif. Chaque plan de l'image tend à s'autonomiser comme s'il était indépendant des autres. Ajout, suppression, répétition du Même ou de variantes posent les fondements du film. Le personnage de Betty naît de cette image et de ce contexte, son visage se dessinant fragilement en surimpression, baigné de lumière. Bien que Betty n'apparaisse pas parmi les danseuses - elle est déjà une autre, ou plutôt une série d'autres -, elle semble faire face à un public, alors que des applaudissements retentissent. Ce prologue assume également une fonction narrative dans la mesure où Diane affirme que son succès lors d'un concours de danse l'a incitée à se lancer dans une carrière d'actrice. Le désir de l'héroïne s'origine donc dans une prestation associée au simulacre et à l'aliénation de l'individu dans une masse indifférenciée. "Tout le monde peut être une star»: tel est l'imaginaire, récemment réactivé par de nombreuses émissions TV 14,
14 Voir sur ce point Laurent Guido, "L'usine aux icônes. Notes sur Popstars, Siegfried Kracauer et son "ornement de la masse'", in HorsChamp, $\mathrm{n}^{\circ}$ 8, printemps/été 2002, p. 58-61. Le jingle de l'émission Popstars basé sur l'interchangeabilité de personnages réduits à des formes graphiques en mouvement s'apparente au prologue de Mulholland Drive. Le casting auquel se présente la première Camilla poursuit cette représentation d'une exhibition standardisée, le play-back renvoyant à la même aliénation de l'individu. 
15 André Gardies, "Esquisse pour un portrait sémiologique de l'acteur ", Le conteur de l'ombre, Editions Aléas, Lyon, 1999.

16 Ainsi, le fait que Naomi Watts et Laura Helena Harring se soient surtout illustrées à la télévision rappelle le projet initial de Lynch, et intervient dans l'opposition cinéma/télévision qui parcourt le film au niveau esthétique. En outre, Naomi Watts a été engagée par Lynch alors qu'elle venait de terminer un court métrage, Ellie Parker, qui raconte, sur un mode comique dont Mulholland Drive pourrait être le pendant tragique, I'histoire d'une femme qui rêve de devenir actrice. D'ailleurs, le réalisateur de ce film, Scott Coffey, qui avait déjà joué dans Lost Highway, apparaît dans Mulholland Drive sous les traits de Wilkins. Comme Lynch aime à s'entourer d'une même équipe, confiant de petits rôles à certains de ses collaborateurs, le procédé de répétition/variation que l'on constate pour ses personnages principaux sont parfois également valables en ce qui concerne le choix de certains acteurs qui incarnent des personnages secondaires, voire de simples figurants.

17 Lorsque A. J. Greimas développe dans Sémantique structurale (PUF, Paris, 1970, p. 172-189) la démarche de Vladimir Propp qui consistait à dégager des constantes d'un ensemble de récits (en l'occurrence des contes russes merveilleux), il considère I'histoire racontée comme la mise en jeu d'un ensemble de forces qu'il nomme "actants", terme générique qui ne fait pas référence à leurs incarnations particulières. Ces actants sont définis relativement à la fonction dont ils sont investis à l'intérieur d'un système de relations mutuelles que Greimas appelle le "schéma actanciel". II résulte de l'articulation de six composantes: le destinateur, le destinataire, le sujet, l'objet, l'adjuvant et l'opposant. Le fort degré de généralisation offert par ce modèle permet de rendre compte des "abstractions" que Lynch lui-même prétend convoquer dans ses films (voir le dernier entretien de Retour à Mulholland Drive). Par ailleurs, l'actualisation spécifique du schéma n'est pas fixe, mais peut subir des reconfigurations. II est ainsi possible de rendre compte des permutations opérées dans les récits de Lynch.

18 Le diptyque resnaisien, adaptation du "cycle " de pièces Intimate Exchanges d'Alan Ayckbourne, procède également à la juxtaposition de possibles développés au sein d'un récit arborescent dont chaque jonction, établie en fonction du temps diégétique (réitération d'ellipses d'égale amplitude, fixées successivement à 5 jours/ 5 semaines/5 ans), obéit à la logique du "ou qui a bercé les rêves de Betty. La seconde partie du film révèlera le caractère utopique de ce projet en montrant que ces possibles sont soumis à une même impossibilité cristallisée dans l'image du corps en décomposition de Diane/Betty.

En dépit de disparitions subites ou de modifications radicales qui exhibent leur nature construite et composite, les personnages de Lynch constituent des repères essentiels dans la compréhension du récit. L'éclatement qu'ils subissent peut selon moi être appréhendé à l'aune d'un modèle pluristratifié du personnage, celui de la "figure actorielle» proposé par André Gardies 15. Les niveaux considérés par Gardies me semblent convenir particulièrement bien à la discussion du fonctionnement de tels films qui, en procédant à une dissociation des différentes facettes du personnage généralement imbriquées en un tout d'apparence homogène, opèrent dans une visée créatrice une démarche analogue à l'entreprise analytique de Gardies. Le modèle sémiologique de ce dernier comprend, dans un ordre croissant d'abstraction, les quatre niveaux suivants: le comédien, être de chair et de sang auquel peuvent être associés d'autres films dans lesquels il a joué 16 , voire une partie de son vécu; le personnage, élément de l'univers diégétique identifié par un patronyme fictionnel; le rôle, renvoi intertextuel à un ensemble de codes propres à un genre; enfin l'actant, c'est-à-dire la position occupée par le personnage au sein du «schéma actanciel» greimasien ${ }^{17}$. Dans les deux films de Lynch, comme dans Smoking / No Smoking (1993) d'Alain Resnais 18 ou dans Cet obscur objet du désir (1977) de Luis Bunuel 19, l'effacement du comédien derrière le(s) personnage(s) qu'il incarne est enrayé par une logique de dissociation entre ces deux niveaux de la figure actorielle: Patricia Arquette est Renée et Alice dans Lost Highway, Naomi Watts et Laura Helena Harring incarnent successivement Betty et Rita, puis Diane et Camilla dans Mulbolland Drive, alors que trois personnages (Fred/Pete et l'individu nommé «Mystery Man» au générique de fin), interprétés par trois acteurs fort différents, semblent n'en faire qu'un seul dans Lost Highway. Dans les sillages des ces variations, d'autres personnages périphériques subissent également des modifications. Les interactions entre les différentes instances de la figure actorielle sont exploitées par Lynch comme de véritables générateurs de récit.

\section{Les seuils de nouveaux possibles}

Lost Highway et Mulholland Drive connaissent tous deux un point de basculement où s'opère une redistribution des données diégétiques, notamment les attributs des personnages et, par conséquent, les différentes composantes de la figure actorielle. Dans Mulholland Drive, cette 
césure est d'autant plus surprenante pour le spectateur qu'elle intervient après ce qui constitue la durée standard d'un long métrage de fiction (env. 1h50'). C'est significativement après la mise en scène, au club "Silencio", du dévoilement du statut illusoire de la représentation que se déroule la séquence-pivot de la boîte bleue à l'issue de laquelle l'innocente Betty devient Diane la vengeresse. Dans cette séquence, Betty et Rita rentrent côte à côte comme deux sœurs - Rita porte d'ailleurs la perruque blonde modelée sur la coupe de cheveux de Betty -, proches et unies pour la dernière fois. Rita possède la clé bleue qu'elle avait trouvée dans son sac à main, alors qu'elle y cherchait avec Betty un indice de son identité; Betty vient de découvrir, également dans son sac à main (lieu de l'intimité féminine), la boîte que cette clé semble pouvoir ouvrir. L'alliance des deux objets mènera à la dissociation du couple, visualisé par un double escamotage des protagonistes, puis par la disparition des objets eux-mêmes. Lorsque la clé réapparaîtra dans la seconde partie du film, elle aura une apparence beaucoup plus réaliste: la grosse clé en plastique qui ressemble à un jouet d'enfant deviendra une clé de consigne courante, comme on en trouve dans nombre de films policiers. L'éviction successive des deux personnages féminins, tabula rasa nécessaire à un nouveau départ, s'opère sans coupe apparente. En effet, Betty disparaît durant un plan-séquence, la caméra qui l'avait rejetée hors-champ ne la retrouvant plus lorsqu'elle retourne à son point de départ; Rita, quant à elle, semble s'être évanouie lors d'un zoom avant vers l'intérieur de la boîte dont l'obscurité, qui occupe l'entièreté de l'écran, masque le changement de plan (fig. 2-5). La forte

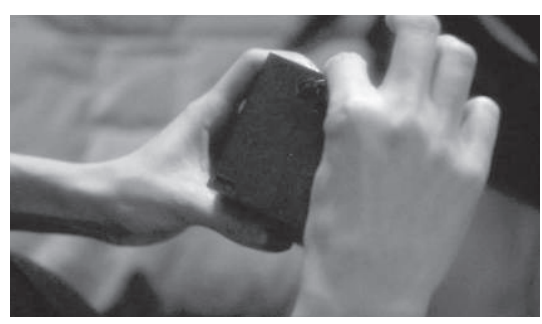

2

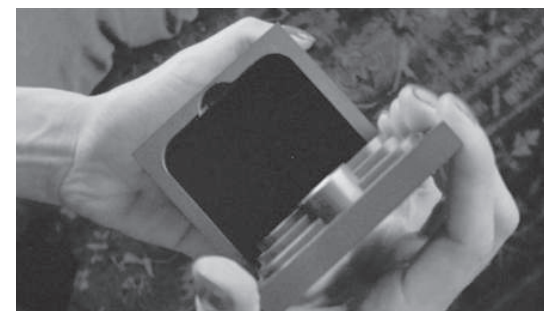

3

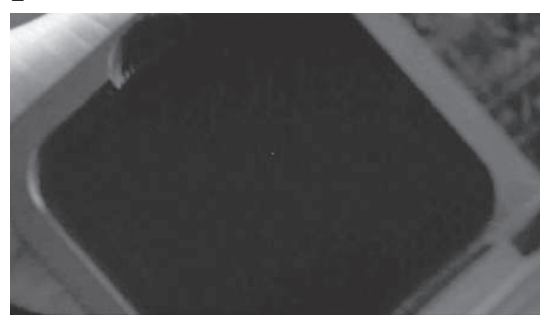

4 bien...". Toutefois, contrairement aux films de Lynch où une certaine ambiguïté est maintenue, ce fonctionnement narratif est donné comme tel au spectateur, qui y prend un plaisir ludique tout en se voyant poussé à évaluer de manière comparative les incidences des différentes décisions des personnages sur l'orientation de leur destin. Lost Highway et Mulholland Drive, en jouant de l'indécision du statut du personnage, renouent plutôt avec les interférences de niveaux de réalité à l'œuvre (de manière certes plus complexe) dans L'année dernière à Marienbad de Resnais (1961). Par contre, le foisonnement de versions établies à partir de données narratives identiques s'accompagne dans Smoking / No Smoking d'une multiplication des personnages joués par les deux seuls interprètes des deux films: Sabine Azéma incarne cinq personnages, Pierre Arditi quatre. Comme chez Lynch, la récurrence de lieux identiques constitue une fonction structurante d'importance. Néanmoins, contrairement à Mulholland Drive, chaque personnage y est individualisé par une série d'attributs stylisés (en adéquation avec la facticité des décors) qui touche différents paramètres comme la voix, l'âge, la démarche, etc.; chez Lynch, Rita se distingue peu, sur le plan physique, de Camilla, et Betty ressemble beaucoup à Diane. Les différences sont plutôt le résultat de l'influence des revers du destin.

19 Dans cette adaptation de La femme et le pantin de Pierre Louÿs, co-écrite avec Jean-Claude Carrière, deux actrices incarnent les versants d'un seul et même personnage appelé Conchita, sans qu'aucun autre personnage de la diégèse ne soit conscient des substitutions: Carole Bouquet représente la froide distinction "à la française", Angela Molina l'extravagance quelque peu vulgaire "à l'espagnole" (l'apparence et l'origine des actrices ne sont donc pas aléatoires). Aucune hiérarchie n'est instaurée entre les deux actrices, comme le suggèrent les mentions du générique où les deux noms apparaissent successivement dans un ordre inversé. David Lynch utilise donc un procédé narratif inverse: au lieu d'avoir deux actrices pour un personnage, Mulholland Drive et Lost Highway donnent deux rôles à une même actrice. Alors que, chez Bunuel, une certaine unicité diégétique persiste (il s'agit de l'expression métaphorique d'un personnage scindé, paradoxal), les deux films de Lynch s'ouvrent sur des possibles. "Cet obscur objet du désir" est toutefois une expression qui s'applique également à ces derniers: le caractère insaisissable de l'être désiré est à la source des transformations qu'il subit. Cette situation s'y complique toutefois du fait que le sujet désirant (Betty/Diane; Fred/Pete) est lui-même soumis à des variables. 
20 Fred semble se demander à qui appartient ce bras qu'il sent sur son dos alors qu'il fait l'amour avec sa femme. "Jamais tu ne m'auras!" lui lance Alice, le double de Renée, après leur étreinte dans le sable. Ainsi, le gros plan sur le bras nu de Renée renvoie-t-il à une présence physique qui n'appartient pas vraiment au corps de l'être désiré. Dans le bref flash qui révèle l'image refoulée par Fred de l'assassinat de sa femme, on entr'aperçoit un corps démembré, ainsi qu'un bras ensanglanté qui gît sur la blancheur des draps du lit conjugal. La fragmentation du montage s'est alors transmise avec violence à l'action du film.

21 Twin Peaks (Twin Peaks, Fire Walk with me, 1992) met en œuvre une modalité encore différente avec la disparition de l'agent Chester Desmond: alors qu'il s'apprête à saisir une bague aperçue sous une caravane, il "subit" un arrêt sur image, puis s'évanouit dans un fondu au noir. Lorsque sa disparition est évoquée par les agents du FBI, l'interpénétration des mondes est introduite par une superposition d'images (voir à propos de ce passage l'article de François Bovier et André Chaperon dans la présente livraison).

$\mathbf{2 2}$ Cette scène, avec ses fumigènes, ses éclairs et ses lumières colorées, rappelle les laboratoires de création d'êtres artificiels dans les films fantastiques. La référence à ce genre renvoie ainsi à un autre possible interprétatif.

23 C'est aussi au gré d'une avancée dans les ténèbres que l'on se fraye un passage à travers l'orifice du masque de John Merrick pour "raccorder" avec l'espace de la chaufferie de l'hôpital (Elephant Man, 1980), que l'on pénètre par une suite de fondus dans la sombre cavité de l'oreille arrachée de Blue Velvet ou que I'on se rapproche jusqu'à l'aveuglement de la couverture du premier plan post-générique de Mulholland Drive. Cette première image de Mulholland Drive constitue d'ailleurs un argument-clé de l'interprétation onirique du film. Georgy Katzarov (L'invention du rêveur, op. cit., p. 4) la commente en ces termes: "L'énonciation du film est ainsi articulée. Elle jaillira du continuité induite par les mouvements d'appareil (comparativement au montage) rend ces disparitions particulièrement étranges, car le spectateur croit partager la même temporalité que les personnages, puis prend soudainement conscience qu'il n'a pas subi la "saute» qui les affecte. Ce principe est fondamentalement différent de celui qui prévaut dans Lost Highway, où la substitution s'insinue systématiquement entre les plans. Par exemple, c'est au gré d'un contrechamp que, soudain, Renée apparaît à Fred avec le visage mortifère de l'Hommemystère. Lost Highway joue sur la fragmentation, visualisation concrète de l'inaccessibilité de l'objet du désir et de l'impuissance à le satisfaire. Le récit est voué à une répétition infinie parce que Fred/Pete ne réussit jamais à "posséder» sa conjointe qui lui échappe toujours, en dépit des changements identitaires 20.

Le passage d'un monde à l'autre s'effectue sur le mode de la suture dans Lost Highway et du flux dans Mulholland Drive 21. Les deux films font par contre usage d'une même figure, déjà évoquée à propos de la scène de la boîte bleue: le plan noir.

Juste avant que Fred ne se transforme en Pete dans la prison 22, la lampe de sa cellule s'éteint, comme si elle menaçait de provoquer l'arrêt du projecteur même du film. De l'obscurité émerge la route du générique que nous empruntons à toute allure jusqu'à ce que nous nous arrêtions devant le nouveau personnage de Pete, debout au bord de la route, éclairé par les phares de la voiture que nous sommes censés occuper (fig. 6-8). Ce trajet nocturne et imaginaire fait office de transition entre les deux personnages. Il sera suivi de plus de vingt secondes d'une image non figurative montrant une sorte de lueur jaunâtre sur un fond noir, puis de quelque seize secondes d'image totalement noire. Lynch utilise fréquemment l'image noire pour nous faire passer d'un monde à l'autre ${ }^{23}$, mais Lost Highway, particulièrement travaillé dans sa dimension plastique, en fait l'utilisation la plus radicale. Dans la scène de prison, les noirs endossent dans un premier temps la fonction traditionnelle d'intercalaires entre différents moments considérés comme itératifs; ensuite, ils s'autonomisent et pourraient correspondre à ce que le personnage «voit» intérieurement (ou, précisément, ce qu'il ne peut ou ne veut voir),

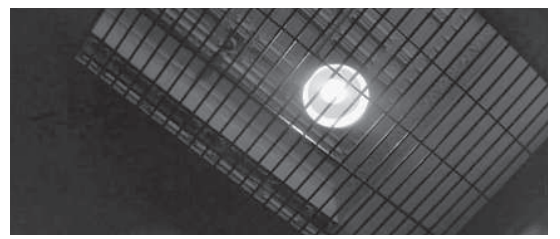

6

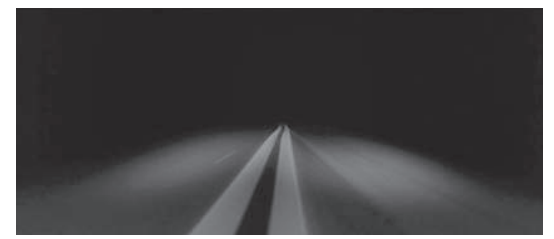

7

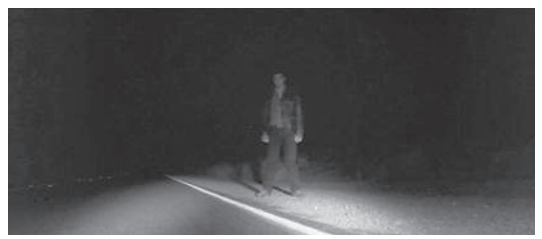

8 

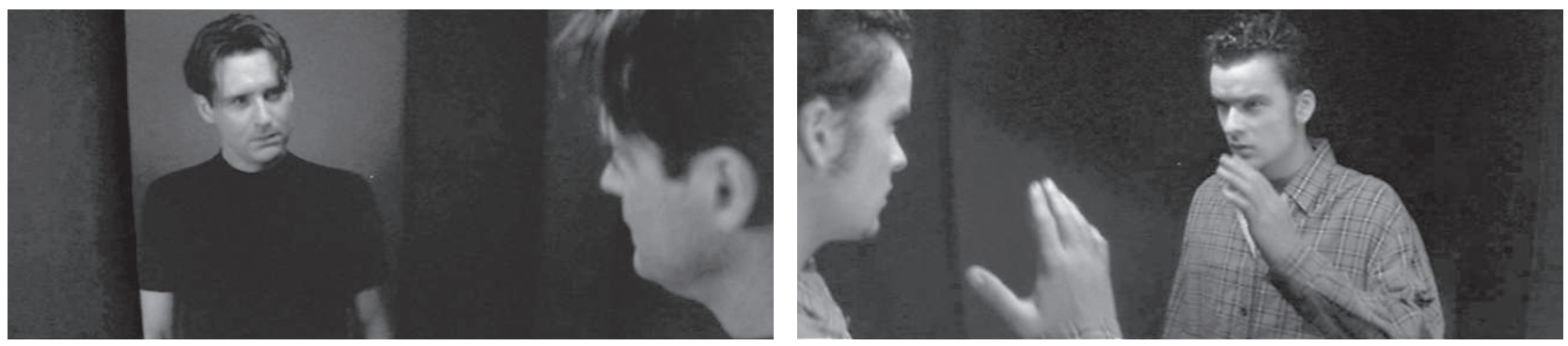

tout en continuant de fonctionner comme ponts interséquentiels. Chez Lynch, le noir se fait le point de convergence de l'univers diégétique et de sa mise en forme filmique : il est à la fois «procédé de ponctuation » $\mathbf{2 4}$ et partie intégrante de l'environnement des personnages.

Lost Highway est un film hanté par la peur du noir. Les recoins obscurs de l'appartement de Fred et Renée sont autant d'espaces soustraits à toute cartographie du réel. Lorsque Fred disparaît dans l'ombre, on ne sait s'il va s'y perdre ou s'il ressortira avec le visage d'un autre. Pourtant, c'est lui-même qu'il rencontre dans le miroir qui émerge de l'obscurité comme du néant (fig. 9), mais cette image se répétera (inversée, le personnage se trouvant alors à gauche de l'écran) dans la seconde partie, lorsque Pete, la nuit tombée, palpe son visage devant la glace comme s'il s'agissait de celui d'un autre (fig. 10). Après une dizaine de secondes de noir complet, un autre "miroir» nous est révélé par un zoom arrière: il s'agit de l'écran du téléviseur sur lequel les époux visionnent les images vidéo qu'un intrus a filmées chez eux à leur insu, représentations objectivées de l'une des facettes du Moi de Fred qui, lors de la révélation du meurtre au spectateur, feront office d'images subjectives.

Au-delà de leur signification conventionnellement symbolique (la «face cachée» du Dr. Jekyll), ces noirs participent à l'insertion invisible mais sensible de non-dits, de lacunes et de refoulements. La non-vision, qui suspend momentanément la nécessité propre au médium de donner une apparence physique aux personnages, permet toutes les mutations, tous les tours de passe-passe. Le noir est le lieu d'origine du double, il annonce l'imminence d'un personnage en devenir. Dans Mulholland Drive, l'accident nocturne mène à l'opacité totale de l'amnésie, qui touche également Pete dans Lost Highway puisqu'il ne sait rien de "l'autre nuit» dont ses parents craignent de lui parler, avant qu'ils ne disparaissent définitivement à l'issue d'une discussion téléphonique entre Pete et l'Homme-mystère. Les conditions de leur disparition me noir. De ce noir-là, d'un visage plongé dans l'étoffe". Toutefois, là où la literie fait écran, nous ne pouvons être sûrs de rien. Katzarov rapporte cette énonciation à un personnage (Diane), alors qu'il peut aussi bien s'agir d'une marque de la méga-énonciation du film, d'un narrateur qui nous guide dans d'obscurs méandres.

24 Au sens de Christian Metz (voir "Ponctuations et démarcations dans le film de diégèse", Essais sur la signification au cinéma, tome II, Klincksieck, Paris, 1972, p. 111-137). 
25 Dans La voix au cinéma (Editions de l'Etoile / Cahiers du Cinéma, Paris, 1982, chapitre I), Michel Chion discute les pouvoirs particuliers (ubiquité, omniscience, omnivoyance) généralement conférés à ces personnages "d'acousmêtres " qui appartiennent à la diégèse tout en n'étant pas visualisés, du moins pour un temps (celui de leur règne). L'acousmêtre maléfique inspiré du Dr. Mabuse de Fritz Lang est récurrent chez Lynch depuis Sailor et Lula. Ses films sont hantés par la menace souterraine d'une organisation secrète au statut ambigu, à michemin entre la mafia, la franc-maçonnerie et une figuration allégorique de processus inconscients.

26 Bernadette Bensaude-Vincent et Christine Blondel (Des savants face à l'occulte 18701940, Editions de la Découverte, Paris, 2002, p. 10-11) soulignent à propos de ce paradigme

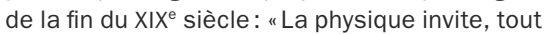
autant que la psychologie, à incorporer l'occulte dans le champ de la science normale. Les techniques nouvelles, le téléphone, puis surtout la télégraphie sans fil, ne permettentelles pas la transmission, instantanée et à distance, des paroles et des pensées?". Lynch fait interagir les sphères du psychique et de la physique. conduisent à aborder brièvement un troisième point relatif aux modalités du passage entre les mondes.

La négation de la vision imposée par les plans noirs trouve en effet une transposition diégétique dans la représentation de la technologie du son, par exemple le téléphone. L'écoute à distance ou l'enregistrement "phonographique» implique une disparition de la source de la voix. Celle-ci est alors qualifiée par Michel Chion d' "acousmatique» 25. Cette voix désincarnée correspond à la présence-absence de ces protagonistes en sursis, susceptibles de disparaître ou de se métamorphoser à tout instant. Le don d'ubiquité de l'Homme-mystère qui se fait appeler au téléphone par Fred alors qu'il se trouve à côté de lui s'applique également à d'autres facettes du même personnage: Fred entend à l'interphone une voix (qui s'avérera être la sienne) lui chuchoter que «Dick Laurent est mort»; c'est à la radio que Pete entend le concert de Fred; dans Mulholland Drive, lorsque Rita téléphone chez une certaine Diane en espérant qu'il s'agisse d'elle-même, elle tombe sur la voix d'un répondeur automatique qu'elle ne reconnaît pas. La communication par des moyens technologiques est un facteur de transfert des personnalités qui s'opère de manière totalement invisible, à l'image d'un courant électrique qui se déplace. Juste avant de disparaître sans laisser de trace, l'agent Desmond de Trin Peaks venait de s'approcher d'un poteau téléphonique dont le bourdonnement électrique était amplifié sur la pisteson du film. Lynch réactualise de façon personnelle une conception qui, au début du $\mathrm{XX}^{\mathrm{e}}$ siècle, associait sciences exactes et spiritisme, technologie du son et surnaturel 26.

\section{La blonde et la brune}

Ne pouvant aborder dans le cadre de cet article toutes les combinaisons auxquelles les personnages des deux films de Lynch donnent lieu, je me contenterai de pointer quelques phénomènes emblématiques en mettant l'accent sur les figures de la blonde et de la brune dont Lynch ne cesse d'exploiter, depuis Blue Velvet, le potentiel différentiel et fantasmatique, comme l'avait fait avant lui Hitchcock dans Sueurs froides (Vertigo, 1958). L'opposition ou la fusion de la blonde et de la brune est en effet un point commun entre Lost Highway et Mulholland Drive. Dans le premier, les deux personnages féminins, Renée (l'épouse brune) et Alice (l'amante blonde, située "de l'autre côté du miroir»), sont interprétés par la même actrice, Patricia Arquette; dans le second, deux actrices, l'une blonde (Naomi Watts), l'autre noiraude (Laura Helena Harring), jouent successivement deux personnages, Betty/Diane et Rita/Camilla. Dans Lost Highway, les deux femmes n'existent que dans leur rapport 
au personnage masculin (Fred/Pete) dont elles semblent être une émanation imaginaire (souvenir de la perte ou fantasme d'un recommencement). Le passage de l'une à l'autre s'effectue d'ailleurs autour de la charnière narrative que représente la transformation de Fred en Pete. La brune Sheila, petite amie de Pete, fait office d'intermédiaire, mais elle incarne, comme la Sandy de Blue Velvet, le quotidien, une "normalité» dépourvue de perversion dont Pete se débarrasse vite au profit du crime dans lequel l'embrigade Alice la vamp. Cette focalisation sur le protagoniste masculin implique que le spectateur en sache peu sur Renée. Montrée dans des scènes peu loquaces, elle ne lui apparaît pas beaucoup plus «construite» que Rita l'amnésique. Lynch fait débuter ses deux films avec des personnages "vides" sur lesquels tout peut encore être greffé (ou "projeté») et qui sont appréhendés rétrospectivement au travers de leur double interprété par la même actrice. Toutefois, Lost Highway procède à une permutation de la blonde et de la brune, alors que Mulholland Drive se concentre sur la première.

La cohérence de ces films est notamment assurée par la référence aux lieux, qui font office de repères. Dans Lost Highway, c'est le bar "Moke's» qui tisse un lien entre les deux femmes: Renée déclare à son mari qu'elle y a rencontré Andy, et que c'est à cette occasion qu'il lui a proposé un job à propos duquel elle reste fort vague. Plus tard, Alice propose à Pete de cambrioler la villa d'Andy, ancien "client» et réalisateur de films pornos dont elle a fait la connaissance au "Moke's». Jamais visualisé, ce lieu insaisissable - l'appellation s'apparente d'ailleurs au terme "smoke", la fumée étant un motif visuel récurrent chez Lynch renvoie au passé brumeux des personnages. Le fast-food «Winkie's» de Mulholland Drive, désigné par écrit dans un plan d'ensemble où l'on aperçoit son enseigne (fig. 11) puis sur le badge d'une serveuse (fig. 12) - il est associé à la mythique rue des stars, "Sunset Blvd", et donc au film noir homonyme de Billy Wilder - joue un rôle identique de carrefour des possibles. Betty, qui y mange avec son amie Rita, remercie la serveuse appelée Diane alors que, plus tard dans le film, la même Naomi Watts, assise au même endroit mais cette fois sous le nom de

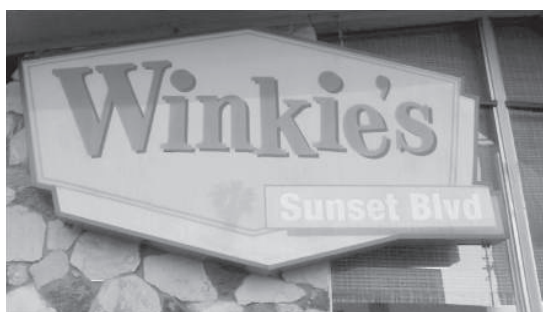

11

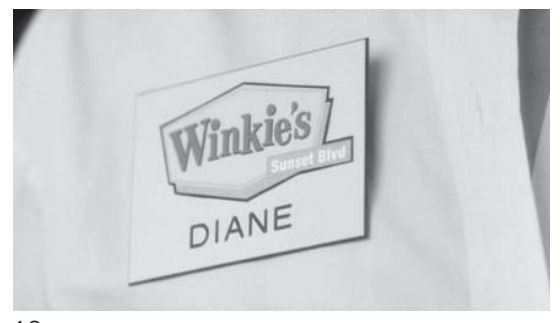

12 


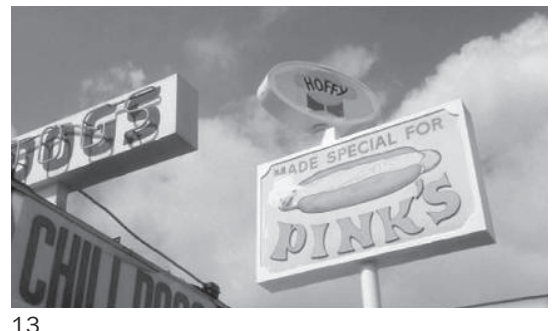

13

27 Plus loin dans le film, Diane croise cet homme, debout à côté de la caisse du Winkie's au moment même où le tueur évoque la clé bleue associée à l'assassinat. Cette interpénétration des pistes narratives accrédite la thèse de la culpabilité refoulée proposée dans le film Retour à Mulholland Drive.

28 En faisant primer le temps diégétique sur le temps du récit, les analyses qui visent à "remettre dans l'ordre" les diverses actions du film font très souvent l'impasse sur le travail de montage qui entrelace de façon minutieuse les différentes pistes narratives.

29 Sur la différence entre le $v u$ et le lu au cinéma, voir Roger Odin, "L'entrée du spectateur dans la fiction ", in Jacques Aumont, JeanLouis Leutrat (éd.), Théorie du film, Albatros, Paris, 1980 , p. 202-205.
Diane (la serveuse se prénommant alors Betty), engagera un tueur pour se débarrasser de Camilla. C'est aussi dans ce restaurant que se déroule la scène périphérique de l'homme confiant à un interlocuteur son effroi devant un clochard monstrueux tapi derrière le bâtiment, scène qui peut se comprendre comme un déplacement de la relation Betty/Rita (la première rassurant la seconde en proie à une crise identitaire), mais aussi comme une manifestation de l'angoisse de Betty/Diane 27. Par ailleurs, ce fast-food connaît un petit frère dont l'appellation présente une forte ressemblance phonétique avec le "Winkie's", et renvoie également à l'une des couleurs dominantes des scènes de "film dans le film»: il s'agit du "Pink's", restaurant dont l'enseigne mobile apparaît dans le premier plan (fig. 13) d'une brève séquence au cours de laquelle le tueur demande à une prostituée junkie de l'informer si une nouvelle recrue se montre sur le trottoir. Il est important de noter que cette séquence devant le "Pink's» est insérée au milieu de la discussion entre Betty et Rita qui fait suite à la découverte de la clé bleue. Cet emplacement $\mathbf{2 8}$ renforce l'association entre la présence de la clé et les événements liés au tueur, et souligne leur fonction de médiation dans la relation Betty/ Rita. Toutefois, l'insertion n'intervient pas entre le champ sur Betty et son contrechamp sur Rita: de part et d'autre de la séquence du "Pink's", la caméra fixe Betty. Cette mise en évidence du personnage interprété par Naomi Watts pourrait insinuer un lien entre Betty et la prostituée, qui est également blonde et dont le look (jeans et T-shirt gris délavé) sera celui de Diane à partir du moment où Camilla se refusera à elle. Cette variante nous inciterait à considérer ce personnage secondaire comme un avatar de la provinciale qui rêvait de faire carrière à Hollywood, mais que la désillusion a poussé au tapin et à la drogue. Ce possible n'est toutefois pas véritablement actualisé par le film, notamment par le fait que la prostituée n'est pas nommée. On l'a compris, l'onomastique constitue un facteur essentiel de l'organisation narrative de Lost Highway et de Mulholland Drive. Toponymes et anthroponymes sont omniprésents dans les répliques des personnages, mais aussi sous forme écrite, ce qui accentue leur prégnance, le lire se distinguant de la modalité dominante de la perception cinématographique (à l'exception des génériques) 29. Certes, l'écrit désigne, individualise, authentifie parce qu'il est un élément fixe et récurrent. Toutefois, ce qui varie chez Lynch dans le cas des noms propres de personnages, c'est leur référent. Rien n'y est plus arbitraire que le signe. "Betty" n'est pas le nom juste pour qualifier le personnage qu'incarne Naomi Watts, mais juste un nom, pourraiton dire en déplaçant l'objet de la formule godardienne de l'image au verbal. Les noms propres, qui sont pourtant, sur le plan linguistique, 
des «désignateurs rigides» $\mathbf{3 0}$ qui ne devraient subir aucune variation, acquièrent dans les fictions de Lynch le statut strictement fonctionnel d'étiquettes que le cinéaste colle et décolle pour mieux coller son spectateur. Le personnage est le nom, il se réduit à lui.

Parallèlement, les autres facettes de la figure actorielle entrent dans la danse des transformations. L'actant-sujet /Diane/ ne vise plus, comme Betty, à découvrir l'identité de sa compagne, mais à la faire abattre : elle est donc également le destinateur et le destinataire de cette funeste entreprise. Alice, contrairement à Renée qui était littéralement «l'objet» de Fred, occupe également une place d'actant-sujet relativement à l'intrigue du cambriolage. La position actancielle de Camilla (sujet dans la relation de vouloir avec l'objet /carrière/, opposant du sujet /Diane/ relativement au même objet) est induite par son rôle qui est annoncé dès la première partie de Mulholland Drive, puisque l'amnésique en fuite s'approprie le nom qui figure sur une affiche de film, «Rita» (pour «Rita Hayworth» dans Gilda, le film réalisé par Charles Vidor en 1946) 31. Les prénoms en «-a», suggérés ou actualisés dans un personnage «réel» (Rita, Camilla) ou fictif (Sylvia, Gilda) dans la diégèse, se multiplient pour renvoyer de manière diffractée à la figure archétypale de la «femme fatale» - et au genre cinématographique du «film noir» auquel elle est rattachée - qui contribuera à construire la Camilla de la seconde partie. L'amnésie de Rita est déjà une expression symbolique de cet archétype, la «femme fatale» incarnant celle dont on ne sait rien, l'Inconnu par excellence. Il faut à la «femme sans nom» un dispositif réflexif pour qu'elle adopte le prénom «Rita»: l'affiche du film Gilda apparaît en effet dans un petit miroir amovible situé à côté du miroir mural de la salle de bains dans lequel se mire l'amnésique (fig. 14). Un zoom avant dans l'axe du plus petit miroir se fait l'expression visuelle de l'opération de substitution, car il fait sortir progressivement Rita du champ (fig. 15), celui-ci étant alors envahi par l'image doublement secondarisée de Rita Hayworth (l'image peinte de l'affiche et son reflet dans le miroir). La star prend la place du personnage sans nom, mais apparaît dans une image inversée qui convient bien à la première partie du film où le rôle de la «femme fatale»
30 Voir Saul Kripke, La logique des noms propres, Minuit, Paris, 1982 [1980].
31 Le fait que l'amnésique choisisse le prénom de la star et non celui du personnage éponyme de Gilda est un élément révélateur du jeu opéré par Mulholland Drive sur la figure actorielle. Par ailleurs, Richard Dyer ("Resistance through Charisma: Rita Hayworth and Gilda" in Women in Film Noir, British Film Institute, Londres, 1978, p. 91-99) a justement montré qu'une particularité du film de Vidor réside dans le fait que la star Rita Hayworth, grâce à la présence qu'elle impose, tend à infirmer la représentation archétypale de séductrice maléfique à laquelle le regard masculin de Johnny (Glenn Ford) ne cesse de l'associer (notamment par le biais de la voix over). II se peut bien que David Lynch ait été sensible à ce divorce entre l'interprète et le rôle qui, selon Dyer, trouve son expression la plus explicite dans les deux interprétations de la chanson Put the Blame on Mame, une situation d'auto-mise en scène du personnage que I'on trouve fréquemment chez Lynch.

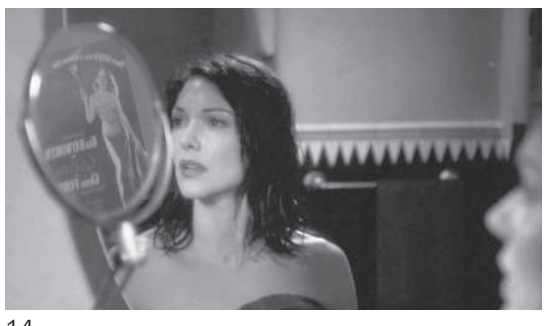

14

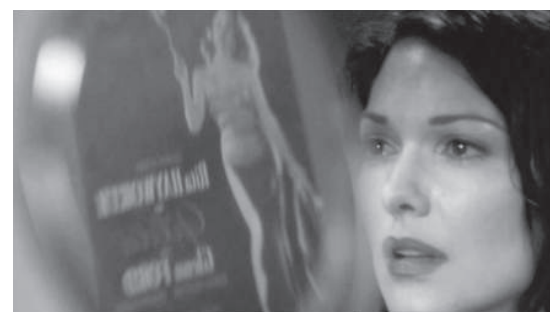

15 
32 Une confusion est entretenue à ce sujet, puisque les plans rapprochés dans la salle de bains, notamment sur une paire de ciseaux, semblent faire penser que Betty a coupé et teint les cheveux de Rita. Cette ambiguïté momentanée, qui se résout peu après lorsque Rita prend place dans le lit de Betty, me semble renvoyer à la superposition des possibles, mais aussi au fait que l'opposition blonde/brune demeure un invariant quel que soit le possible actualisé. est suggéré tout en étant nié par les attitudes de Rita, naïve et soumise à son amie qui la guide. Plus tard, après la découverte du cadavre d'une blonde, Rita repousse l'issue tragique associée au rôle de manipulatrice qu'elle a endossé au travers de son identification au film Gilda, et prend Betty comme modèle, demandant à celle-ci de l'aider à ajuster une perruque $^{32}$. Or, lorsque les deux femmes se regardent dans la même glace murale que celle évoquée précédemment, le miroir amovible a disparu: il est remplacé par Betty qui se tient sur la gauche de l'écran (fig. 16).

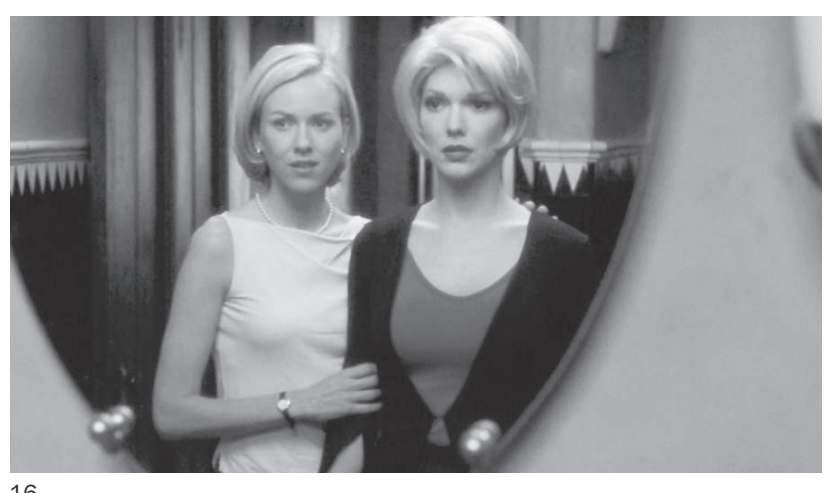

16

Rôles, fonctions, attributs des personnages et choix des interprètes sont soumis à un principe de circulation qui crée diverses combinatoires entre le nom et l'apparence physique. L'usage de la photographie en témoigne: chez Andy, Pete découvre une image noir/blanc sur laquelle Renée et Alice posent, debout entre Andy et l'énigmatique Mr. Eddy (fig. 17). Or, tout est affaire de point de vue: lorsque les agents de police observent cette même image sur le lieu du crime, elle ne montre qu'un seul personnage féminin, la brune Renée (fig. 18). Une femme (brune) peut en cacher une autre (blonde). Pour le spectateur, ces agents livrent des repères utiles à la compréhension du changement de monde : ce sont eux qui reconnaissent dans Mr. Eddy le fameux «Dick Laurent» (qui est plus une expression autonome qu'un personnage), un nom qui résonne, paré de mystère et associé à la mort, depuis le début du film.

Dans Mulholland Drive, la photographie est liée à l'expression qui la désigne ("this is the girl!»), répétée à trois reprises: le mafieux l'utilise pour imposer l'actrice blonde en présentant au réalisateur Adam une photographie noir/blanc qui porte la légende "Camilla Rhodes»; le cinéaste reprend cette formule pour exprimer son choix de l'actrice (toute désignée pour le rôle) lors du casting; enfin, Diane prononce la même phrase lorsqu'elle tend une photo similaire au tueur, mais, cette 
fois, l'image montre le visage de Laura Helena Harring. Le plan est cadré de sorte à laisser hors-champ une partie de la légende, le spectateur devant se contenter de lire les cinq lettres "ODHES» pour en inférer le nom "(Camilla R)odhes».

Lost Highway et Mulholland Drive reposent pour une grande part sur des interactions aléatoires entre des mots et des images qui, en convoquant des phénomènes intertextuels, complexifient les films en leur ouvrant d'autres possibles. Le spectateur est invité à un jeu sur les mots qui, heureusement, se prolonge au-delà de la vision du film.

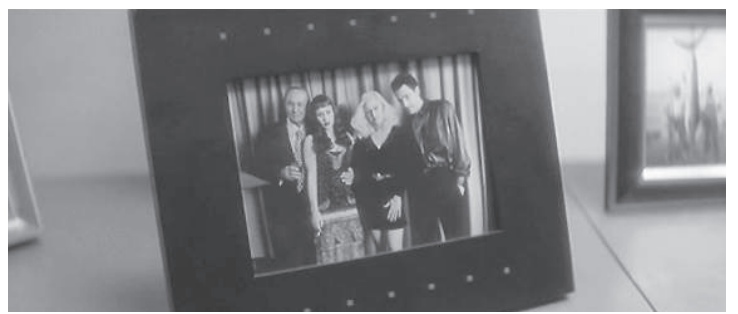

17

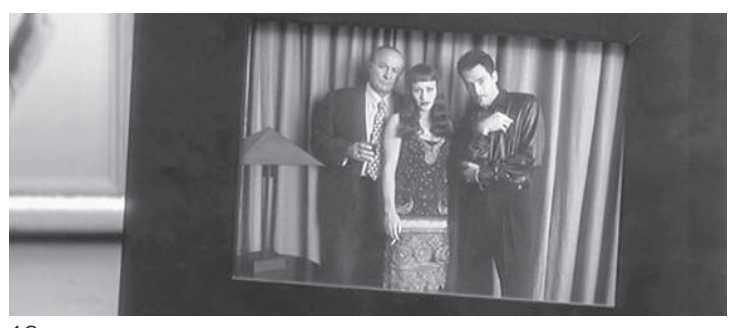

